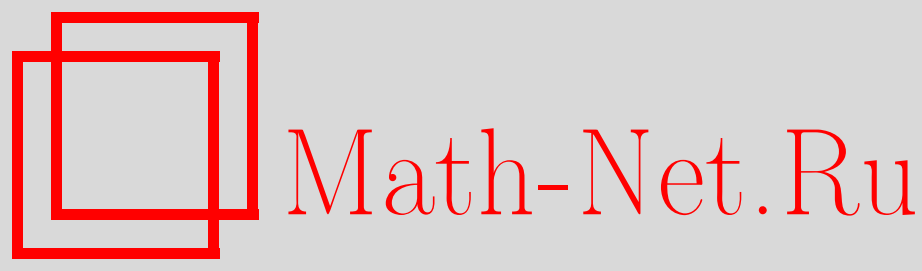

Е. Е. Тареева, Ю. Д. Фомин, Е. Н. Циок, В. Н. Рыжов, Закритические аномалии и линия Видома для изоструктурного фазового перехода в твердом теле, ТМФ, 2018, том 194, номер 1, 175-184

DOI: https://doi.org/10.4213/tmf9386

Использование Общероссийского математического портала Math-Net.Ru подразумевает, что вы прочитали и согласны с пользовательским соглашением http://www.mathnet.ru/rus/agreement

Параметры загрузки:

IP : 34.229 .45 .116

26 апреля 2023 г., 16:25:00

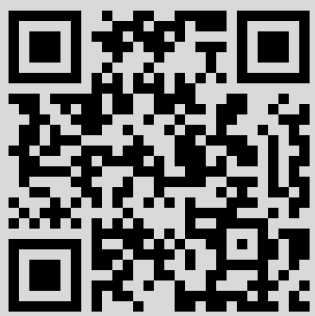




\title{
ЗАКРИТИЧЕСКИЕ АНОМАЛИИ И ЛИНИЯ ВИДОМА ДЛЯ ИЗОСТРУКТУРНОГО ФАЗОВОГО ПЕРЕХОДА В ТВЕРДОМ ТЕЛЕ
}

\begin{abstract}
Представление о линии Видома как линии максимумов корреляционной длины и ряда термодинамических функций отклика выше критической точки было введено для описания аномалий, наблюдаемых в воде выше гипотетической критической точки перехода жидкость-жидкость. Закритическая область при переходе газ-жидкость также была позже описана в терминах линии Видома. Естественно предположить, что в закритической области для изоструктурного перехода первого рода в кристаллах, заканчивающегося в критической точке, также существует аналог линии Видома. Для исследования свойств новой линии Видома использована простая полуфеноменологическая модель, близкая по духу теории Ван-дер-Ваальса. Вычислены термодинамические функции отклика выше критической точки изоструктурного перехода и найдены их максимумы, определяющие положения линии Видома.
\end{abstract}

Ключевые слова: изоструктурный переход, закритическая область, аномалии термодинамических величин, линия Видома.

DOI: https://doi.org/10.4213/tmf9386

\section{1. ВВЕДЕНИЕ}

Хорошо известно, что простая теория Ван-дер-Ваальса (см., например, книги [1], [2]) вполне удовлетворительно (во всяком случае, на качественном уровне) описывает простые жидкости и переход жидкость-газ, включая критическую точку, в терминах точных аналитических выражений, позволяющих проследить физическую картину ряда явлений. Недавно в рамках исходной классической теории Ван-дер-Ваальса была описана аналитическим путем и закритическая область жидкости [3], [4]. В настоящей работе мы проводим исследование подобного типа для области термодинамических параметров твердого тела, находящейся за критической

Работа поддержана Российским научным фондом (грант № 14-22-00093).

${ }^{*}$ Институт физики высоких давлений им. Л.Ф. Верещагина РАН, Москва, Россия. E-mail: etare@ms2.inr.ac.ru 
точкой окончания изоструктурного фазового перехода первого рода. В такой критической точке также сходятся три фазы одной (в данном случае кристаллической) симметрии. В основе нашего расчета лежит простая допускающая аналитическое решение модель, предложенная в работе [5].

В последние десятилетия сильно возрос интерес к исследованию поведения сверхкритической (закритической) жидкости - состояния, находящегося за критической точкой $\left(T_{\mathrm{c}}, P_{\mathrm{c}}\right)$, в которой заканчивается переход жидкость-газ первого рода. Это связано прежде всего с тем, что лишь недавно в эксперименте удалось достичь области температур и давлений, в которой находится закритический флюид для большинства веществ. При этом оказалось, что закритические флюиды - очень хорошие растворители и интересны с точки зрения промышленных технологий в химии и фармакологии [6]. Что касается теоретических аспектов физики закритического флюида, они интересны и сами по себе. Наиболее важными задачами здесь являются описание термодинамического поведения флюида вблизи критической точки и исследование изменения динамики при понижении температуры [7].

В критической точке расходится корреляционная длина $\xi$ для термодинамических флуктуаций [8]. В закритической области свойства флюида меняются непрерывным образом, без сингулярностей, но физические величины, определяемые вторыми производными термодинамического потенциала Гиббса, ведут себя весьма сложно, проходя через максимумы и образуя систему “гребней” (ridges), сходящихся к критической точке. Такими величинами являются, например, изотермическая сжимаемость $\beta_{T}$, коэффициент теплового расширения $\alpha_{P}$, теплоемкость $C_{P}$. Линия, на которой лежат максимумы корреляционной длины и виртуально должны были бы лежать все указанные максимумы, поскольку все указанные величины связаны с корреляционной длиной, была названа линией Видома в статье Стенли с соавторами [9]. На самом деле, как было показано недавно, ее длина, т. е. область, где линии максимумов совпадают, составляет доли от $T_{\mathrm{c}}$ и $P_{\mathrm{c}}$. Можно говорить о линии Видома и для перехода жидкость-жидкость. Оба типа линии Видома интенсивно исследуются в последнее время (см. работы [3], [7], [9]-[23]).

Как нам представляется, понятие закритической области можно распространить и на твердое тело. Мы имеем в виду такие системы, в которых при изменении температуры или давления происходит изоструктурный фазовый переход первого рода. При этом имеет место скачок объема, но кристаллографическая структура твердого тела не меняется. При определенных изменениях внешних параметров скачок объема уменьшается и линия сосуществования фаз заканчивается в некоторой точке, которую естественно назвать критической точкой. За критической точкой остается та же кристаллографическая структура. Таким образом, в этой точке, как и в привычной критической точке, заканчивающей линию перехода газ-жидкость, сходятся три фазы с одной и той же симметрией.

Изоструктурный фазовый переход уже давно был обнаружен в некоторых реальных простых металлах (например, в церии и его соединениях). Позднее в более тонких экспериментах некоторые из результатов были поставлены под сомнение. По-видимому, наиболее достоверным можно считать наличие такого перехода в цирконии [24]. Но основные исследования этого феномена относятся к более позднему времени (см. статьи [25]-[27]) - речь идет о компьютерном моделировании коллоидных систем. Для потенциала их взаимодействия характерно отталкивание типа твердой сферы и притяжение, глубину и радиус действия которого можно менять как в реальном, так и в компьютерном эксперименте. 


\section{2. ПОСТАНОВКА ЗАДАЧИ И МОДЕЛЬ}

Результаты компьютерного моделирования коллоидных систем с изоструктурным переходом были достаточно успешно объяснены в рамках теоретических подходов в ряде работ [5], [28]-[32]. В результате этих исследований стало ясно, что изоструктурный переход в твердом теле имеет место в том случае, когда ширина области, где взаимодействие носит характер притяжения, является очень малой (не более 0.07 от ширины отталкивания), а глубина ее - значительной. Оказалось, что именно для таких “экстремальных" [28] потенциалов (почти “липких сфер”) характерен изоструктурный переход с критической точкой в твердом состоянии. Качественными отличиями изоструктурного перехода в твердом теле от перехода жидкость-газ являются существенная асимметрия (относительно плотности) изоструктурного перехода в окрестности критической плотности и зависимость характерной ширины притягивающей части потенциала от его конкретной формы.

Подчеркнем, что закритическую область для изоструктурного фазового перехода в твердом теле до сих пор никто не рассматривал.

При исследовании закритической области для изоструктурного перехода мы развиваем подход, основанный на результатах статьи [5], в которой для твердого тела была предложена полуфеноменологическая теория типа теории Ван-дер-Ваальса. Здесь мы напоминаем основные положения этой теории и переходим затем к изложению наших результатов.

В основе теории лежит использование вариационного принципа Боголюбова для свободной энергии $F$ возмущенной системы с гамильтонианом $H$ :

$$
F \leqslant F_{1}=F_{0}+\left\langle\left(H-H_{0}\right)\right\rangle_{0} .
$$

Здесь $F_{0}$ - свободная энергия невозмущенной системы с гамильтонианом $H_{0}$. Для приближенной свободной энергии мы используем нижнюю грань оценки.

Главную роль в обычной теории Ван-дер-Ваальса играет отталкивание частиц. Мы так же в качестве исходной системы со свободной энергией $F_{0}$ и гамильтонианом $H_{0}$ возьмем простейшую систему с отталкивательным взаимодействием твердых сфер $V_{0}(r)=\epsilon \phi_{\mathrm{HS}}(x)$, где $x=r / \sigma$, а $\sigma$ - диаметр твердой сферы:

$$
\begin{aligned}
& \phi_{\mathrm{HS}}(x)=\infty, \quad x \leqslant 1, \\
& \phi_{\mathrm{HS}}(x)=0, \quad x>1 \text {. }
\end{aligned}
$$

Притягивающую часть взаимодействия $V_{\mathrm{A}}(r)$ будем рассматривать как поправочный член и положим

$$
\begin{aligned}
\phi_{\mathrm{A}}(x) & =0, & & x \leqslant 1, \\
\phi(x) & =-\frac{1}{x^{n}}, & & x>1 .
\end{aligned}
$$

Кроме того, в соответствии с духом теории Ван-дер-Ваальса пренебрежем корреляциями в области вне твердых сфер. Это означает, что поправочный член $\Delta F$ в свободной энергии можно приблизить следующим образом:

$$
\int d \mathbf{r}_{1} \int d \mathbf{r}_{2} \rho_{2}^{\mathrm{HS}}\left(\mathbf{r}_{1}, \mathbf{r}_{2}\right) V_{\mathrm{A}}\left(r_{12}\right) \simeq \int d \mathbf{r}_{1} \int d \mathbf{r}_{2} \rho_{1}^{\mathrm{HS}}\left(\mathbf{r}_{1}\right) \rho_{1}^{\mathrm{HS}}\left(\mathbf{r}_{2}\right) V_{\mathrm{A}}\left(r_{12}\right),
$$


где $\rho_{1}(\mathbf{r})$ и $\rho_{2}\left(\mathbf{r}_{1}, \mathbf{r}_{2}\right)$ - одночастичная и двухчастичная функции распределения соответственно.

Для идеального кристалла с узлами решетки $\mathbf{r}_{j}$ можно записать

$$
\rho_{1}^{\mathrm{HS}}(\mathbf{r})=\sum_{j=1}^{N} \varphi\left(\mathbf{r}-\mathbf{r}_{j}\right),
$$

где $\varphi\left(\mathbf{r}-\mathbf{r}_{j}\right)$ - нормированное на единицу распределение плотности вокруг узла $\mathbf{r}_{j}$. Считая это распределение $\delta$-функцией и переходя к суммам по оболочкам, а затем оставляя лишь ближайших соседей, получаем после всех упрощений соотношение

$$
\Delta f \equiv \frac{\Delta F}{N \epsilon}=-\frac{1}{2} n_{1} \phi\left(x_{1}\right),
$$

где $n_{1}$ - число ближайших соседей, а $x_{1}$ - приведенное расстояние до ближайшего соседа.

Что касается свободной энергии для системы тведых сфер, то она получается с помощью уравнения состояния с использованием приближения, аналогичного приближению свободного объема для жидкости. Теперь оно заменяется приближением свободного пробега. Введем величину $\delta$ такую, что $V^{1 / 3}\left(1-\delta^{1 / 3}\right)$ представляет собой среднее расстояние, которое может свободно пройти твердая сфера в объеме $V$ кристалла из твердых сфер. Здесь $\delta=\rho / \rho_{\text {ср }}$, где $\rho_{\text {ср }}-$ плотность плотнейшей упаковки для данной структуры. Теперь

$$
\frac{\beta p_{\mathrm{HS}}(\rho)}{\rho}=\frac{1}{1-\delta^{1 / 3}} \simeq \frac{3 \rho_{\mathrm{cp}}}{\rho_{\mathrm{cp}}-\rho} .
$$

Здесь $\beta=1 / T$. Учитывая далее, что можно считать $x_{1}=\left(\rho_{\mathrm{cp}} / \rho\right)^{1 / 3}$, и принимая во внимание, что $p=-\partial f / \partial v$, получаем окончательно из формул (3) и (4) уравнение состояния твердого тела в виде

$$
\frac{p}{\rho_{\mathrm{cp}} \sigma^{3}}=\frac{t}{x_{1}^{2}\left(x_{1}-1\right)}+\frac{n_{1} \phi^{\prime}\left(x_{1}\right)}{6 x_{1}^{2}},
$$

где $t=n_{1} T$.

Как уже говорилось, с помощью изложенного выше подхода в работе [5] был описан собственно изоструктурный переход и построены фазовые диаграммы для нескольких потенциалов. Закритическая область не рассматривалась.

\section{3. ЗАКРИТИЧЕСКАЯ ОБЛАСТЬ ДЛЯ ИЗОСТРУКТУРНОГО ПЕРЕХОДА В ТВЕРДОМ ТЕЛЕ}

Перейдем теперь к исследованию закритической области. Основное уравнение (5) аналогично уравнению Ван-дер-Ваальса для перехода газ-жидкость, поэтому в рамках данного подхода невозможно рассчитать корреляционные функции, в частности, невозможно вычислить корреляционную длину - величину, лежащую в основе определения линии Видома. Однако, имея в своем распоряжении уравнение состояния (5), мы можем с помощью простых формул аналитическим путем получить выражения для ряда термодинамических величин, максимумы которых должны 
лежать на линии Видома. Речь идет о функциях отклика, определяемых вторыми производными термодинамического потенциала.

В работах по компьютерному моделированию коллоидных систем наиболее часто встречается изоструктурный переход между двумя (более плотной и более рыхлой) фазами гранецентрированной кубической решетки. Далее мы рассматриваем именно этот случай. Это значит, что в (5) мы должны положить для числа ближайших соседей $n_{1}=12$, а для максимальной плотности наиплотнейшей упаковки твердых сфер считать $\rho_{\text {ср }}=\sqrt{2}$. Будем измерять давление в единицах $\rho_{\text {ср }} \sigma^{3} n_{1}$, т. е. введем величину

$$
P=\frac{p}{\rho_{\mathrm{cp}} \sigma^{3} n_{1}}
$$

Теперь уравнение состояния (5) в случае потенциала $1 / r^{n}$ (в окончательных расчетах мы положим $n=100)$ принимает вид

$$
P=\frac{t}{12 x^{2}(x-1)}-\frac{n}{6 x^{n+3}}
$$

где нижний индекс у $x_{1}$ мы не пишем. Приведенная плотность $z=\rho / \rho_{\text {ср }}$ связана с $x$ соотношением $x=z^{-1 / 3}$.

Найдем критическую точку $z_{\mathrm{c}}, t_{\mathrm{c}}$, в которой заканчивается изоструктурный переход. Она дается решением системы уравнений

$$
\frac{\partial P}{\partial z}=0, \quad \frac{\partial^{2} P}{\partial z^{2}}=0
$$

Используя равенство (6), получаем, что $z_{\mathrm{c}}=0.94335, t_{\mathrm{c}}=1.0524$.

Рассмотрим теперь поведение некоторых термодинамических величин в закритической области вблизи критической точки.

Используя известную формулу для изотермической сжимаемости

$$
\beta_{T}=-\frac{1}{V}\left(\frac{\partial V}{\partial P}\right)_{T}=\frac{1}{z}\left(\frac{\partial z}{\partial P}\right)_{T},
$$

выводим из (6) соотношение

$$
\beta_{T}=\frac{18 x^{n+3}(x-1)^{2}}{(t / 2) x^{n+1}(3 x-2)-n(n+3)(x-1)^{2}} .
$$

На рис. 1 приведены графики зависимостей изотермической сжимаемости от плотности $z$ вдоль изотерм при температурах $t=1.06,1.08,1.1$.

Положение линии максимумов на изотермах описывается уравнением

$$
t=2 \frac{n(n+3)^{2}(x-1)^{3}}{x^{n+1}\left(9 x^{2}-11 x+4\right)} .
$$




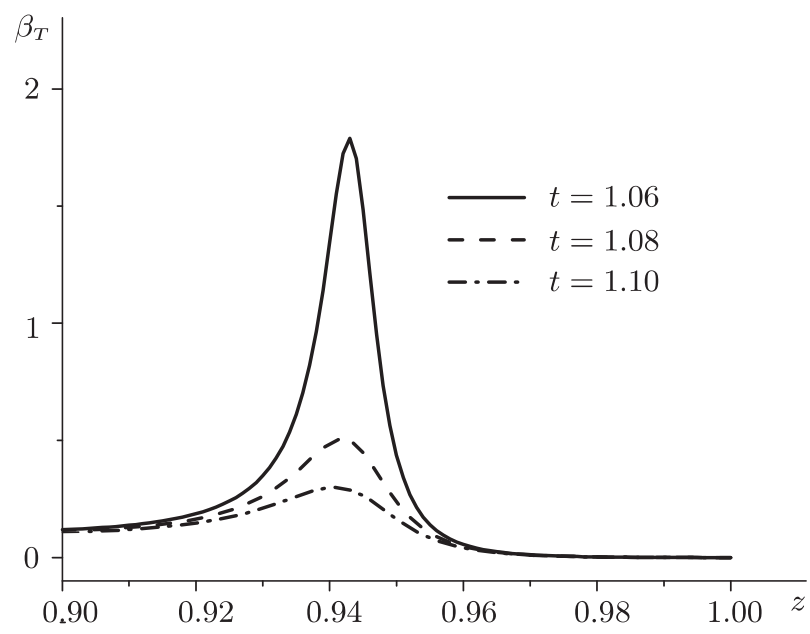

Рис. 1. Поведение изотермической сжимаемости $\beta_{T}$ как функции плотности на изотермах $t=1.06,1.08,1.1$.

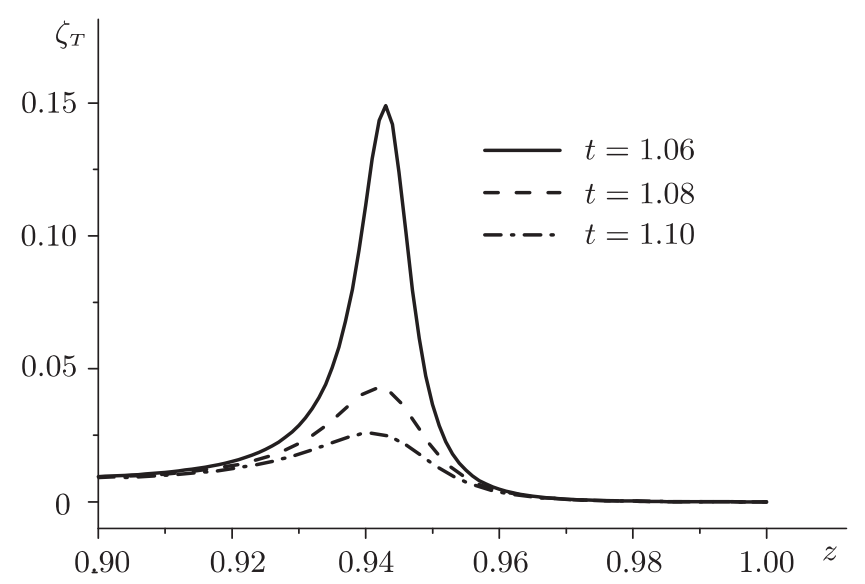

Рис. 2. Поведение величины флуктуаций плотности $\zeta_{T}$ как функции плотности на изотермах $t=1.06,1.08,1.1$.

С помощью выражения (6) нетрудно получить величину флуктуаций плотности $T(\partial z / \partial P)_{T}=\zeta_{T}$ :

$$
\zeta_{T}=\frac{(3 t / 2) x^{n}(x-1)^{2}}{(t / 2) x^{n+1}(3 x-2)-n(n+3)(x-1)^{2}} .
$$

На рис. 2 приведены кривые $\zeta_{T}(z)$ при значениях температуры $t=1.06,1.08,1.1$ в закритической области. Кривая максимумов на изотермах описывается равенством

$$
t=\frac{n^{2}(n+3)(x-1)^{3}}{x^{n+1}(2 x-1)} .
$$




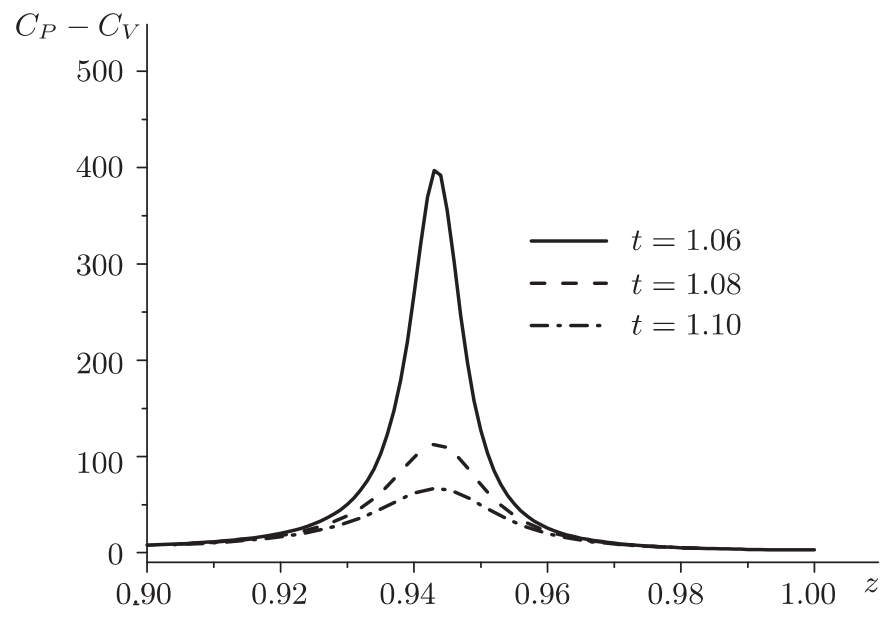

Рис. 3. Поведение теплоемкости $C_{P}-C_{V}$ как функции плотности на изотермах $t=1.06,1.08,1.1$.

На рис. 3 для тех же значений температуры приведены кривые теплоемкости

$$
C_{P}-C_{V}=-T \frac{(\partial P / \partial T)_{V}^{2}}{(\partial P / \partial V)_{T}}=\frac{(3 t / 2) x^{n+2}}{(t / 2) x^{n+1}(3 x-2)-n(n+3)(x-1)^{2}} .
$$

Линия максимумов на изотермах описывается уравнением

$$
t=\frac{n(n+3)[2-n(x-1)](x-1)}{x^{n+1}} .
$$

Аналогично, для коэффициента теплового расширения $\alpha_{P}$ (см. рис. 4) записываем

$$
\alpha_{P}=-\frac{1}{V}\left(\frac{\partial V}{\partial T}\right)_{P}=\frac{18 x^{n+1}(x-1)}{(t / 2) x^{n+1}(3 x-2)-n(n+3)(x-1)^{2}},
$$

уравнение для линии максимумов имеет вид

$$
t=2 \frac{n(n+3)[n(x-1)-1](x-1)^{2}}{x^{n+2}} .
$$

Из рис. 1-4 видно, что рассмотренные термодинамические величины в закритической области изоструктурного перехода так же, как в закритическом флюиде (см. работы [3], [4], [7], [15]), образуют гребни максимумов, которые быстро размываются при удалении от критической точки. Можно сказать, что длина линий максимумов в твердом закритическом теле еще короче, чем в закритическом флюиде [3], [4], [7].

На рис. 5 приведены кривые $t(z)$, на которых лежат максимумы величин $\beta_{T}, \zeta_{T}$, $C_{P}-C_{V}$ и $\alpha_{P}$ на изотермах в области, расположенной непосредственно вблизи критической точки $t_{\mathrm{c}}, z_{\mathrm{c}}$, построенные соответственно по формулам (8)-(14). Из рис. 5 видно, что гребни расходятся, как и в случае флюида [3], [7], [15], [16] (и даже быстреe), так что "линия Видома" имеет почти нулевую длину. Рядом расположены лишь линии для $\beta_{T}$ и $\zeta_{T}$. 


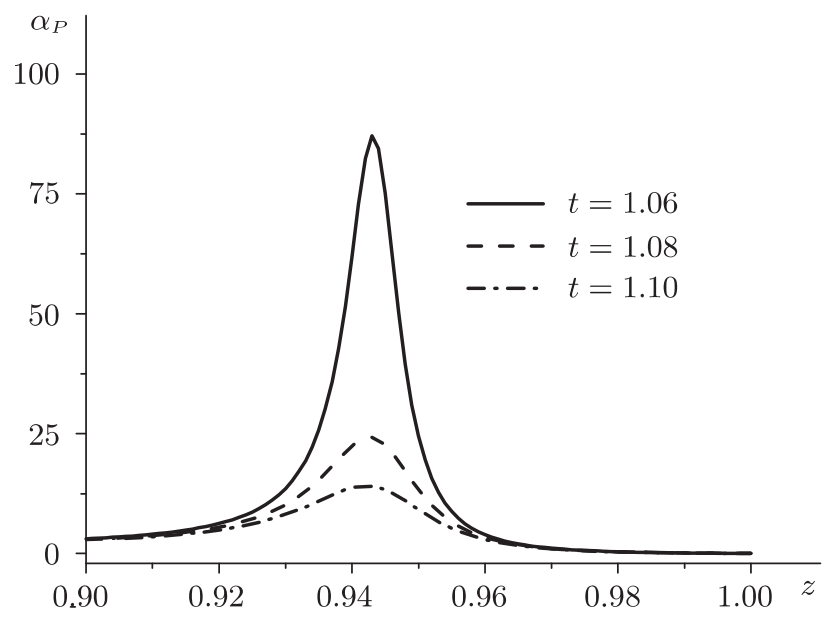

Рис. 4. Поведение коэффициента теплового расширения $\alpha_{P}$ как функции плотности на изотермах $t=1.06,1.08,1.1$.

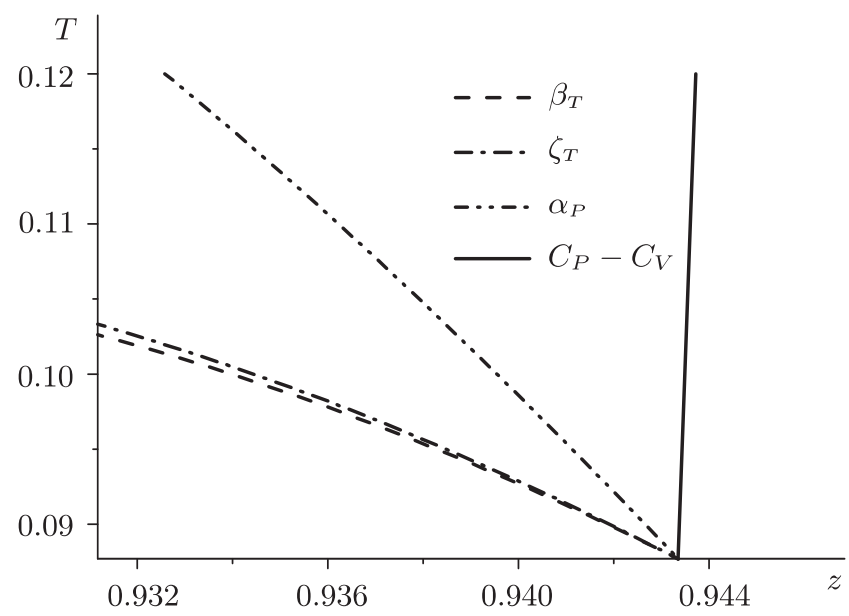

Рис. 5. Линии максимумов величин $\beta_{T}, \zeta_{T}, C_{P}-C_{V}$ и $\alpha_{P}$ на изотермах на плоскости $t-z$.

Из рис. 5 следует еще один интересный вывод. Порядок следования линий максимумов в закритическом твердом теле таков же, как в закритическом флюиде [3], [7], [15], [16].

\section{4. ЗАКЛЮЧЕНИЕ}

Проведено изучение физических характеристик закритической области для изоструктурного фазового перехода в твердом теле. Эта область, лежащая выше критической точки, в которой заканчивается фазовый переход первого рода между двумя фазами (более плотной и менее плотной) с одинаковой кристаллической симметрией, рассмотрена впервые. 
В рамках простой модели, которая построена исходя из представлений статистической механики с привлечением приближений, являющихся обобщением основных положений теории Ван-дер-Ваальса на случай твердого тела, в закритической области вблизи критической точки рассчитаны следующие термодинамические функции отклика: изотермическая сжимаемость, величина флуктуаций плотности, теплоемкость и коэффициент теплового расширения.

Как и в случае закритического флюида, эти величины образуют систему максимумов, практически исчезающих уже на небольшом расстоянии от критической точки. Порядок следования линий максимумов такой же, как в закритическом флюиде.

Благодарности. Авторы благодарны В. В. Бражкину за полезные обсуждения. Работа была выполнена с использованием оборудования центра коллективного пользования "Комплекс моделирования и обработки данных исследовательских установок мега-класса" НИЦ “Курчатовский институт”, http://ckp.nrcki.ru/.

\section{Список литературы}

[1] Р. Балеску, Равновесная и неравновесная статистическая механика, т. 1, 2, Мир, М., 1978.

[2] J. P. Hansen, I. R. McDonald, Theory of Simple Liquids, Academic Press, New York, 1986.

[3] V.V. Brazhkin, V. N. Ryzhov, "Van der Waals supercritical fluid: exact formulas for special lines", J. Chem. Phys., 135:8 (2011), 084503.

[4] V. V. Brazhkin, V. N. Ryzhov, "Erratum: 'Van der Waals supercritical fluid: exact formulas for special lines"', J. Chem. Phys., 145:5 (2016), 059901.

[5] A. Daanoun, C. F. Tejero, M. Baus, "Van der Waals theory for solids", Phys. Rev. E, 50:4 (1994), 2913-2924.

[6] Supercritical Fluids. Fundamentals and Applications, Nato Science Series E, 366, eds. E. Kiran, P. G. Debenedetti, C. J. Peters (eds.), Springer, Dordrecht, 2000.

[7] В. В. Бражкин, А. Г. Ляпин, В. Н. Рыжов, К. Траченко, Ю. Д. Фомин, Е. Н. Циок, "Где находится область сверхкритического флюида на фазовой диаграмме?", УФН, 182:11 (2012), 1137-1156.

[8] Г. Стенли, Фазовые переходы и критические явления, Мир, М., 1973.

[9] L. Xu, P. Kumar, S. V. Buldyrev, S.-H. Chen, P. H. Poole, E. Sciortino, H. E. Stanley, "Relation between the Widom line and the dynamic crossover in systems with a liquid-liquid phase transition", Proc. Natl. Acad. Sci. USA, 102:46 (2005), 16558-16562.

[10] P. H. Poole, S. R. Becker, F. Sciortino, F. W. Starr, "Dynamical behavior near a liquid-liquid phase transition in simulations of supercooled water", J. Phys. Chem. B, 115:48 (2011), 14176-14183.

[11] G. Franzese, H. E. Stanley, "The Widom line of supercooled water", J. Phys.: Condens. Matter, 19:20 (2007), 205126, 16 pp.

[12] P. F. McMillan, E. H. Stanley, "Fluid phases: going supercritical", Nature Phys., 6 (2010), 479-480.

[13] G. G. Simeoni, T. Bryk, F. A. Gorelli, M. Krisch, G. Ruocco, M. Santoro, T. Scopigno, "The Widom line as the crossover between liquid-like and gas-like behaviour in supercritical fluids", Nature Phys., 6 (2010), 503-507.

[14] F. A. Gorelli, T. Bryk, M. Krisch, G. Ruocco, M. Santoro, T. Scopigno, "Dynamics and thermodynamics beyond the critical point", Sci. Rep., 3 (2013), 1203, 5 pp.

[15] V. V. Brazhkin, Yu. D. Fomin, A. G. Lyapin, V. N. Ryzhov, E. N. Tsiok, "Widom line for the liquid-gas transition in Lennard-Jones System", J. Phys. Chem. B, 115:48 (2011), $14112-14115$. 
[16] V. V. Brazhkin, Yu. D. Fomin, V. N. Ryzhov, E. E. Tareyeva, E. N. Tziok, "True Widom line for a square-well system", Phys. Rev. E, 89:4 (2014), 042136.

[17] P. Gallo, D. Corradini, M. Rovere, "Fragile to strong crossover at the Widom line in supercooled aqueous solutions of NaCl", J. Chem. Phys., 139:20 (2013), 204503.

[18] G. Ruppeiner, A. Sahay, T. Sarkar, G. Sengupta, "Thermodynamic geometry, phase transitions, and the Widom line", Phys. Rev. E, 86:5 (2012), 052103, 4 pp.

[19] H.-O. May, P. Mausbach, "Riemannian geometry study of vapor-liquid phase equilibria and supercritical behavior of the Lennard-Jones fluid", Phys. Rev. E, 85:3 (2012), 031201, 9 pp.; Erratum 86 (2012), 059905.

[20] Yu. D. Fomin, V. N. Ryzhov, E. N. Tsiok, V. V. Brazhkin, "Thermodynamic properties of supercritical carbon dioxide: Widom and Frenkel lines", Phys. Rev. E, 91:2 (2015), 022111, 5 pp.

[21] Y. D. Fomin, V. N. Ryzhov, E. N. Tsiok, V. V. Brazhkin, K. Trachenko, "Dynamic transition in supercritical iron", Sci. Rep., 4 (2014), 7194, 5 pp.

[22] Yu. D. Fomin, V. N. Ryzhov, E. N. Tsiok, V. V. Brazhkin, "Dynamical crossover line in supercritical water", Sci. Rep., 5 (2015), 14234, 6 pp.

[23] Е. Е. Тареева, В.Н. Рыжов, "Закритическая жидкость частиц с потенциалом Юкавы: новое приближение для прямой корреляционной функции и линия Видома", ТМФ, 189:3 (2016), 464-476.

[24] H. Xia, A. L. Ruoff, Y.K. Vohra, "Temperature dependence of the ?-bcc phase transition in zirconium metal", Phys. Rev. B, 44:18 (1991), 10374-10376.

[25] P. Bolhuis, D. Frenkel, "Prediction of an expanded-to-condensed transition in colloidal crystals", Phys. Rev. Lett., 72:14 (1994), 2211-2214.

[26] P. Bolhuis, D. Frenkel, "Isostructural solid-solid transition in crystalline systems with short-ranged interaction", Phys. Rev. E, 50:6 (1995), 4880-4890.

[27] D. Frenkel, "Colloidal encounters: a matter of attraction", Science, 314:5800 (2006), 768-769.

[28] C. F. Tejero, A. Daanoun, H. N. W. Lekkerkerker, M. Baus, "Phase diagrams of 'simple' fluids with extreme pair potentials", Phys. Rev. Lett., 73:5 (1994), 752-755.

[29] C. F. Tejero, A. Daanoun, H. N. W. Lekkerkerker, M. Baus, "Isostructural solid-solid transition of (colloidal) simple fluids", Phys. Rev. E, 51:1 (1995), 558-566.

[30] C. Rascón, L. Mederos, G. Navascués, "Solid-to-solid isostructural transition in the hard sphere/attractive Yukawa system", J. Chem. Phys., 103:22 (1995), 9495.

[31] C. Rascón, L. Mederos, G. Navascués, L. Mederos, "Phase transitions in systems with extremely short-ranged attractions: a density-functional theory", Phys. Rev. B, 51:21 (1995), 14899-14906.

[32] A. R. Denton, H. Löwen, "Isostructural solid-solid transitions in square-shoulder systems", J. Phys.: Condens. Matter, 9:1 (1997), L1-L5. 